\title{
Microlensing Towards the Galactic Bulge
}

\author{
ANDRZEJ UDALSKI, MICHAL SZYMANSKI, JANUSZ KALUZNY, MARCIN \\ KUBIAK \\ Warsaw University, Astronomical Observatory, Al. Ujazdowskie 4, PL-60-286, \\ Warszawa, Poland \\ MARIO MATEO, GEORGE PRESTON, WOJCIECH. KRZEMINSKI \\ Carnegie Observatories, 813 Santa Barbara Street, Pasadena CA, 91101, USA \\ and \\ BOHDAN PACZYNSKI \\ Princeton University Observatory, 124 Peyton Hall, Princeton, NJ, 08544, USA
}

\begin{abstract}
We describe an ongoing survey to search for dark matter via lensing events of stars in the Galactic Bulge. The principal properties of the survey are described, and some preliminary results are shown for newly-discovered variables. We discuss some of the projects related to the study of the Galactic Bulge that can be addressed using these data, and describe the future plans for the survey over the coming few years.
\end{abstract}

Key words: methods: observational - surveys - stars: imaging

Galaxy rotation curves provide convincing evidence for the existence massive halos of unseen material. Paczynski (1986, ApJ, 304, 1) first suggested this dark matter can be detected via gravitational lensing effects if composed of objects more massive than about $10^{-8} \mathrm{M}_{\odot}$. More recently, Paczynski (1991, ApJL, 371, L63) and Griest, et al. (1991, ApJL, 372, L79) noted the significant benefits of searching for microlensing events towards the Galactic Bulge. These include a) the existence of a minimum lensing rate due to low-mass disk stars, b) the presence of a multitude of background sources at a common distance in many different Bulge fields, and c) a reasonably high lensing rate due to putative halo objects. The obvious practical difficulty with this experiment is that only about one star in $10^{6}$ is expected to be 'strongly' lensed (i.e., $\Delta \mathrm{mag} \geq 0.34$ ) at any given time. Thus, millions of stars must be regularly monitored to detect a significant number of events.

We have embarked on a project to conduct a microlensing survey towards the Galactic Bulge. The 1992 observations have been acquired using a $2048 \times 2048$ Ford (Loral) CCD on the $1 \mathrm{~m}$ telescope at Las Campanas Observatory. Fourteen fields were monitored in the $\mathrm{V}$ and I bands, though most were obtained in I. Galaxy clusters have been monitored for SNe when the Bulge is not visible or the seeing is worse than 1.5-1.7 arcsec. The primary survey field is Baade's Window (BW; $\left.l=1^{\circ}, b=-4^{\circ}\right)$; in addition, fields on either side of the Galactic center $(l \sim$ $\pm 5^{\circ}, b \sim-3^{\circ}$ ) were also monitored. Over 1100 I-band images were obtained on $\sim 45$ useful nights between $12 \mathrm{Apr}$ and $10 \mathrm{Aug}$ 1992; an additional $200 \mathrm{~V}$-band images were also acquired.

All analysis was done in near-real-time at the telescope, the photometry being performed using a modified version of the DoPHO'T program developed by Paul Schechter. We were able to reduce about 150,000 stars per hour using two Sparc 2 computers at LCO. The Bulge frames typically contain about 120,000-180,000 measurable stars. Though the reductions are optimized for repeat measurements 
of crowded fields, the quantity and quality of the stellar photometry is nonetheless critically dependent on the seeing. The median seeing for acceptable frames is approximately 1.2 arcsec.

The Figure below shows (uncalibrated) I-band light curves of four newly discovered variables in our survey. Two of these are RR Lyr stars, while the others are different sorts of eclipsing binaries. The Algol variable (star 108954) has a short duty cycle, yet was easily identified with our photometry. The systematic identification of intrinsic variables is important to a) eliminate them from consideration in our survey for lensing events, b) as a source of study in their own right, and c) as a check on the quality of the photometry and our ability to detect variables in our crowded fields.

The 1992 observations were obtained as a pilot project for a more ambitious future survey. We have a) demonstrated the feasibility of monitoring $10^{6}$ stars nightly, b) developed software and techniques to analyze these data in near-realtime, and c) identified many of the intrinsic variable in the Bulge. In the future we plan to pursue a number of projects on the Bulge using the existing data. These include a survey of the age distribution of the Bulge from deep, co-added VI frames of each field and from analysis of the photometric properties of the Algols (Preston, this volume). Also, we plan to study the structure of the Bulge by comparing the distribution of brightnesses of RR Lyr stars in different regions. Our second major goal is to observe more stars, on more nights, with faster and more complete software. We plan to achieve this by applying faster hardware to the survey, constructing a multi-CCD camera, and possibly building a dedicated telescope for the project. Our immediate goals for 1993 are to extend the current survey to more stars in other Bulge fields, and improve our ability to do real-time analysis of the incoming data.
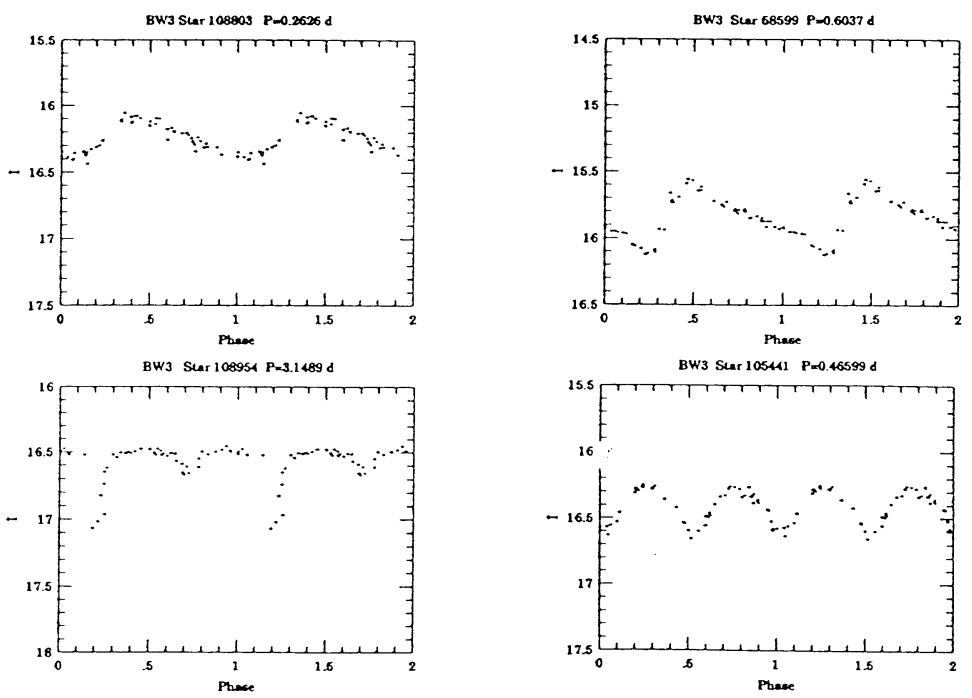

MM acknowledges support through a Hubble Fellowship from STScI under contract to NASA (HF-1007.01-90A). BP acknowledges support from the NSF through grant AST-9023775. 